\title{
Genome-wide Analysis of the CalS gene family in cotton reveals their potential roles in fiber development and responses to stress
}

\author{
Jiajia Feng ${ }^{1,2}$, Yi Chen ${ }^{1}$, Xianghui Xiao ${ }^{2}$, Yunfang Qu ${ }^{1}$, Pengtao $\mathbf{L i}^{2}$, Quanwei Lu ${ }^{\text {Corresp., } 1,2}$, Jinling Huang ${ }^{\text {Corresp. } 1}$ \\ ${ }^{1}$ College of Agriculture, Shanxi Agricultural University, Taigu, Shanxi, China \\ 2 School of Biotechnology and Food Engineering, Anyang Institute of Technology, Anyang, Henan, China \\ Corresponding Authors: Quanwei Lu, Jinling Huang \\ Email address: daweianyang@163.com, huangjlsxau@163.com
}

Callose deposition occurs during plant growth and development, as well as when plants are under biotic and abiotic stress. Callose synthase is a key enzyme for the synthesis of callose. In this study, 27, 28, 16, and 15 callose synthase family members were identified in Gossypium hirsutum, Gossypium barbadense, Gossypium raimondii, and Gossypium arboreum using the sequence of Arabidopsis callose synthase. The CalSs were divided into five groups by phylogenetic, gene structure, and conservative motif analysis. The conserved motifs and gene structures of CalSs in each group were highly similar. Based on the analysis of cis-acting elements, it is inferred that GhCalSs were regulated by abiotic stress. WGD/Segmental duplication promoted the amplification of the CalS gene in cotton, and purification selection had an important function in the CalS family. The transcriptome data and qRT-PCR under cold, heat, salt, and PEG treatments showed that GhCalSs were involved in abiotic stress. The expression patterns of GhCalSs were different in various tissues. We predicted that GhCalS4, which was highly expressed in fibers, had an important effect on fiber elongation. Hence, these results help us understand the role of GhCalSs in fiber development and stress response. 


\section{Genome-wide Analysis of the CalS gene family in cotton reveals their}

\section{2 potential roles in fiber development and responses to stress}

3 Jiajia Feng ${ }^{1,2}$, Yi Chen ${ }^{1}$, Xianghui Xiao ${ }^{2}$, Yunfang $\mathrm{Qu}^{1}$, Pengtao $\mathrm{Li}^{2}$, Quanwei $\mathrm{Lu}^{1,2^{*}}$, Jinling Huang ${ }^{\text {* }}$

$4{ }^{1}$ College of Agriculture, Shanxi Agricultural University, Taigu, Shanxi, China

$5{ }^{2}$ School of Biotechnology and Food Engineering, Anyang Institute of Technology, Anyang, Henan,

6 China

8 * Co-Corresponding Author:

9 Quanwei $\mathrm{Lu}$

10 Anyang, Henan, 455000, China

Email address: daweianyang@163.com

Jinling Huang

Taigu, Shanxi, 030600, China

Email address: huangjlsxau@163.com

\section{Abstract}

Callose deposition occurs during plant growth and development, as well as when plants are under biotic and abiotic stress. Callose synthase is a key enzyme for the synthesis of callose. In this study, 27, 28, 16, and 15 callose synthase family members were identified in Gossypium hirsutum, Gossypium barbadense, Gossypium raimondii, and Gossypium arboreum using the sequence of Arabidopsis callose synthase. The CalSs were divided into five groups by phylogenetic, gene structure, and conservative motif analysis. The conserved motifs and gene structures of CalSs in each group were highly similar. Based on the analysis of cis-acting elements, it is inferred that GhCalSs were regulated by abiotic stress. WGD/Segmental duplication promoted the amplification of the CalS gene in cotton, and purification selection had an important function in the CalS family. The transcriptome data and qRT-PCR under cold, heat, salt, and PEG treatments showed that GhCalSs were involved in abiotic stress. The expression patterns of GhCalSs were different in various tissues. We predicted that GhCalS4, which was highly expressed in fibers, had an important effect on fiber elongation. Hence, these results help us understand the role of GhCalSs in fiber development and stress response.

Keywords. Callose synthase, Synteny, Gene family, Gossypium, various stresses

\section{Introduction}

Callose is a linear homopolymer composed of $\beta$-1, 3-linked glucose, widely found in higher plants as an important part of specialized cell walls or cell wall-associated structures (Chen \& and deposited after overexpression of the AtCalS12 (Ellinger et al., 2013). In rice, callose 
deposition in the plasmodesmata of $\operatorname{crr} 1$ (AtCalS10 homologous gene) mutants was reduced (Song et al., 2016).

Callose can regulate the transport of plasmodesmata and phloem, affect plant development and response to biotic stress by controlling callose synthases. For example, the AtCalS5 could maintain the normal formation of callose walls during pollen development (Shi et al., 2015). Meanwhile, CalS was regulated by various signaling pathways, and different biological regulatory processes involve hormones, transcription factors. For instance, external application of ABA can increase the activity of callose synthase of rice, promoting callose deposition, and thus increasing its resistance to BPH insects (Liu et al., 2017). The expression of CalSs were also affected by developmental and stress conditions, such as pollen development (Toller et al., 2008; Xie et al., 2010; Huang et al., 2009; Shi et al., 2015), low-temperature stimulation (Fromm et al., 2013), mechanical wounding (Cui \& Lee 2016; Jacobs et al., 2003; Xie et al., 2011), fungal diseases (Dong et al., 2008; Oide et al., 2013; Ellinger et al., 2014; Blümke et al., 2013), bacterial diseases (Granato et al., 2019; Enrique et al., 2011), and insect diseases (Ahmad et al., 2011; Koh et al., 2012; Sun et al., 2018; Yao et al., 2020).

Due to the importance of callose, the callose synthase gene family has been identified in various plants. To date, it has been reported as 12 AtCalSs in Arabidopsis thaliana (Hong, Delauney \& Verma, 2001), 12 CsCalSs in Citrus sinensis (Granato et al., 2019), 32 BnCalSs in Brassica napus (Liu et al., 2018), 8 VvCalSs in Vitis vinifera (Yu et al., 2016), 7 HvCalSs in Hordeum vulgare (Schober et al., 2009), 15 BraCalSs in Chinese cabbage (Pu et al., 2019). Generally, according to the evolutionary analysis of CalSs in the above species, the CalS family can be divided into 3 or 4 main groups (Chen \& Kim, 2009; Liu et al., 2018).

Cotton is an important economic crop in China which yield is affected by biotic and abiotic stresses, producing prevalent fibers for textile industry (Wang et al., 2020; Fang et al., 2014). Some researches have reported that cotton fiber elongation was related to callose deposition which may be involved in the closing of plasmodesmata, then promoted the fiber length (Ruan et al., 2004). It is possible that callose affects fiber elongation by controlling CalS. In light of the above, the CalS may play a significant role in cotton in responsing various stress and promoting fiber elongation. A callose synthase gene, CFL1 was identified (Cui et al., 2001), however, the callose synthase gene family members, phylogenetic relationships and expression patterns in cotton are still unclear.

In the current study, we identified callose synthase genes in two cultivated allotetraploids cotton, G. hirsutum and G. barbadense, and their two putative genome donors, G. raimondii and G. arboreum, then discussed their phylogenetic relationships, conserved domains, gene structures, synteny, and cis-acting elements. We also focused on the expression patterns of GhCalSs in various tissues and their expression under abiotic stress. These findings provide a solid foundation for further study of the roles of CalSs in cotton fiber development and stress responses.

\section{Materials \& Methods}

\section{Plant Materials and Treatments.}


Upland cotton TM-1, planted in Anyang Institute of Technology, was subjected to salt stress $(350 \mathrm{mM} \mathrm{NaCl})$ and drought stress (12\% PEG6000) when the seedling reached two weeks. The leaves were collected $0 \mathrm{~h}, 1 \mathrm{~h}, 3 \mathrm{~h}, 6 \mathrm{~h}, 12 \mathrm{~h}$, and $24 \mathrm{~h}$ after treatment. CCRI 45 and MBI7747 were planted on farms managed by Cotton Research of Chinese Academy of Agricultural Sciences in Anyang. Cotton fibers were collected at 5, 10, 15, 20, 25 developmental time-points postanthesis (DPA). All samples were stored at $-80^{\circ} \mathrm{C}$.

\section{Identification of CalS family members in Gossypium spp.}

The genome sequences and annotated files of G. hirsutum (Hu et al., 2019), G. barbadense (Hu et al., 2019), G. raimondii (Paterson et al., 2012), and G. arboretum (Du et al., 2018) were downloaded from Cottongen (https://www.cottongen.org/) (Yu et al., 2014). Both blast and HMMER were used to identify the CalS sequences. The 1, 3-beta-glucan synthase (PF02364) and FKS1_DOM1 domain (PF14288) from the Pfam database (http://pfam.xfam.org/) were searched by the HMMSearch program in TBtools to determine the presumed protein sequence (Chen et al., 2020). Besides, 12 Arabidopsis CalSs (Hong, Delauney \& Verma, 2001) were used as queries sequences to identify family members using the Blastp program of TBtools (Chen et al., 2020). The protein sequences without above two domains were rejected and the domain which incomplete were also deleted. Finally, the final sequences were calculated by using ExPASy (https://www.expasy.org/) to calculate the theoretical isoelectric points (pI) and molecular weights (MW) and using the CELLO (http://cello.life.nctu.edu.tw/) for subcellular localization prediction (Yu et al., 2006).

\section{Phylogenetic tree construction, gene structure, and motif analysis.}

The phylogenetic tree among four Gossypium species and Arabidopsis thaliana was constructed by MEGA7 (Kumar et al., 2016). It was constructed by the neighbor-joining (NJ) method, with 1000 bootstrap replicates, then was drawn by using EvolView (He et al., 2016). TBtools was used to extract the location information of CalSs and visualize the gene structure. MEME (https://meme-suite.org/meme/tools/meme) was used to identify the conservative motif with the parameter set to the maximum number of motifs: 20 .

\section{Chromosome location and synteny analysis for CalSs.}

The locations of CalSs on chromosomes were shown by TBtools using four cotton species genomic annotation files (Chen et al., 2020). MCScanX was used to analyze the collinearity of the CalSs, that is, using CalS protein sequences to analyze the orthologous and paralogous gene pairs (Chen et al., 2020). Collinear gene pairs were visualized by using the circos (Chen et al., 2020). To investigate the selection pressure between homologous genes, we calculated the nonsynonymous substitutions rate $(\mathrm{Ka})$ and synonymous substitutions rate $(\mathrm{Ks})$ of homologous genes by KaKs_Calculator (Wang et al., 2010).

\section{Analysis of Cis-acting element in promoters and functional enrichment analysis.}

The 2000bp sequence upstream of the translation initiation codon ATG of CalS gene was selected as promoter. The cis-acting elements contained in the promoter region of the CalSs were predicted using the PlantCare website (Lescot et al., 2002). For functional enrichment analysis, gene ontology $(\mathrm{GO})$ analysis was performed using the OmicShare tool (https://www.omicshare.com/tools). 


\section{GhCalSs expression pattern under different tissues and abiotic stresses.}

In order to analyze the expression of GhCalSs in different tissues and under stress, we downloaded 11 tissues (bract, pental, torus, root, leaf, stem, pistil, sepal, anther, ovule, fiber) and abiotic stress treatment (cold, heat, drought, salt) data from Cotton Omics Database (http://cotton.zju.edu.cn) (accession number: PRJNA490626) (Hu et al., 2019). GhCalSs with FPKM $>1$ were considered as expressed genes. The expression patterns of the GhCalSs were visualized by ComplexHeatmap (Gu et al., 2016) based on the value of $\log _{2}(\mathrm{FPKM}+1)$.

\section{RNA isolation and qRT-PCR analysis.}

FastPure Plant Total RNA Isolation Kit (RC401, Vazyme) was used to extract RNA, and then we used $1 \mu \mathrm{g}$ to synthesize cDNA (HiScript III 1st Strand cDNA Synthesis Kit, R312 Vazyme). ChamQ Universal SYBR qRT-PCR Master Mix (Q711, Vazyme) was used for qPCR in ABI 7500 Fast Real-time PCR System (Applied Biosystems, USA). Gene-specific primers for qRTPCR were designed by using primer-blast in NCBI, with melting temperatures of $55-60^{\circ} \mathrm{C}$, product lengths of 101-221 bp, primer length of 18-25 bp (Table S1). For qRT-PCR, the reaction contains $10 \mu \mathrm{L} 2 \mathrm{x}$ ChamQ Universal SYBR qPCR Master Mix, $0.4 \mu \mathrm{L}$ of each primer, $3 \mu \mathrm{L}$ template, and $\mathrm{ddH}_{2} \mathrm{O}$ to make up the total $20 \mu \mathrm{L}$ volume. Then it was carried out in the following condition: one cycles of $95^{\circ} \mathrm{C}$ for $30 \mathrm{~s}, 40$ cycles of $95^{\circ} \mathrm{C}$ for $10 \mathrm{~s}$ and $60^{\circ} \mathrm{C}$ for $30 \mathrm{~s}$. Each experiment was repeated three times, and two of the completed data were selected for drawing. Expression of all genes were calculated using a $2^{-\Delta \Delta C t}$ method (Livak \& Schmittgen, 2001).

\section{Results}

\section{Identification and characterization of CalSs in Gossypium spp.}

Through the analysis of the CalS protein sequences in Arabidopsis thaliana, we found that all the protein sequences contain 1, 3- $\beta$-glucan synthase (PF02364) and FKS1_DOM1 domain (PF14288), total of 27 members of the CalS gene family in G. hirsutum, 28 in G. barbadense, 15 in $G$. raimondii, and 16 in G. arboretum were identified, all of which were named according to their chromosomal locations. The properties of CalSs in cotton were further analyzed (Table S2). The protein sequence length of CalSs ranged from 1494 to 1979 amino acids, with an average MW of $212.88 \mathrm{kDa}$, and shared high similarity to the Arabidopsis thaliana CalS proteins (Hong, Delauney \& Verma, 2001). The isoelectric point (PI) values of the above genes were all greater than 7, indicating that CalSs in cotton were alkaline, which was the same as the biochemical properties of the CalSs in Chinese cabbage and Brassica (Pu et al., 2019; Liu et al., 2018). The CalSs were most likely localized in the plasma membrane, as predicted in Arabidopsis thaliana and Chinese cabbage (Pu et al., 2019; Zavaliev et al., 2011).

\section{Classification and phylogenetic analysis of the cotton CalSs.}

In order to investigate the evolutionary relationships of the CalSs in the four cotton species and its relationship with Arabidopsis thaliana, a phylogenetic tree was constructed using the protein sequences of CalSs (Fig. 1). Based on the phylogenetic tree of this study, the CalSs were divided into five groups. The distribution of CalSs in each group was shown in Table S3. The members of Group A were homologous to AtCalS11/AtCalS12, the members of Group B were homologous to AtCalS9/AtCalS10, the members of Group C were homologous to 
159 AtCalS6/AtCalS7, the members of Group D were homologous to AtCalS8, and the members of 160 Group E were homologous to AtCalS1-5. There were Arabidopsis genes homologous to cotton in 161 each group, further indicating that the cotton CalSs and the Arabidopsis thaliana CalSs were 162 close in evolutionary, which was consistent with the evolutionary relationship between 163 Arabidopsis and cotton. It is observed that most of the CalSs derived from At-subgenome of two

\section{cultivated allotetraploids cotton stayed close together with the CalS gene of G. arboretum, and} the CalSs of Dt-subgenome stayed close together with the CalS gene of G. raimondii, which was consistent with the hypothesis that the allotetraploid cotton species were produced by the recombination of two diploid cotton species (Liu et al., 2015). Phylogenetic tree analysis suggested that the CalS homologous gene in cotton may have similar functions.

\section{Gene structure and amino acid motif analysis of the CalSs.}

The diversity of gene structure and differences in conserved motifs are the manifestations of the evolution of multigene families (Magwanga et al., 2018). The distribution of exon/intron regions of CalSs was analyzed to realize the diversity of gene structure (Fig. 2). The number of CalSs exons varied from 1-51, and most CalSs had more than 35 exons (57/86, 66.2\%). Clearly, these CalSs were divided into an exon-poor group ( $<7$ exons, group A) and other exon-rich groups (>37 exons, group B-E) (Fig2, Table S2). The exons of CalSs had high similarity in the same group, and the number of exons in group B, group D, and group E were the same (Fig2, Table S2).

The motif is a conserved region in the sequence (Magwanga et al., 2018). We identified 20 possible motifs using MEME (Fig. 2). Interestingly, all CalSs except GbCalS2/14/15 and GhCalS1 contained motif1-20 and were arranged in the sequence of motif15-9-8-13-12-11-7-2014-16-3-2-1-6-19-5-17-4-18-10. The distribution of CalSs were slightly different among different groups, and only the number and arrangement position were different. The number and arrangement of motifs in the same group of CalSs were more similar than those in other groups.

\section{Chromosomal location, gene duplication, and syntenic analysis of the CalSs in Gossypium spp.}

Based on the sequencing and annotated information of the four cotton genomes, the chromosome length and the distribution of genes on chromosome could be analyzed (Fig. 3). The distribution of CalSs in the two heterotetraploid cotton species chromosomes was highly similar. For example, CalSs had the same number and distribution on chromosomes A03, A04, A05, A08, A11. In G. hirsutum, $27 \mathrm{GhCalSs}$ were distributed on 15 chromosomes, including $13 \mathrm{GhCalSs}$ in At-subgenome and 14 GhCalSs in Dt-subgenome. In G. barbadense, 28 GbCalSs were distributed on 16 chromosomes, including 14 GbCalSs in At-subgenome and 14 GbCalSs in Dtsubgenome. In $\mathrm{G}$. arboreum, $16 \mathrm{GaCalSs}$ were distributed on 8 chromosomes and a scaffold. In G. raimondii, $15 \mathrm{GrCalSs}$ were distributed on 8 chromosomes. Most CalSs occured at the upper or lower arms of chromosomes. D08 and D10 chromosomes both had the largest number of CalSs in the two allotetraploid cotton. Obviously, chromosome length was not positively correlated with the distribution number of CalSs on chromosomes.

Gene duplication is the basis for the functional differentiation of homologous genes, the main reason for the generation of new functional genes (Conant \& Wolfe, 2008). In order to

Peer) reviewing PDF | (2021:08:65037:2:0:NEW 27 Oct 2021) 
200 explain the gene replication events of CalSs in cotton, we identified 15, 14 paralogous gene pairs

201

202

203

204

205

206

207

208

209

210

211

212

213

214

215

216

217

218

219

220

221

222

223

224

225

226

227

228

229

230

231

232

233

234

235

236

237

238

239

240

in G. hirsutum, G. barbadense respectively, and 1 pair in G. arboreum. But there was no paralogous gene pair in G. raimondii (Table S4). GhCalS21/22, GbCalS1/2 as well as GbCalS22/23 were tandem duplication. In the four cotton species, the duplication events of the CalSs were WGD/ Segmental, Tandem Duplicates, Dispersed, and proximal duplication, and the main expansion mechanism was WGD/Segmental (Table S2).

In order to illustrate the collinearity of CalS genes, we analyzed the orthologous and paralogous gene pairs (Fig. 4, Table S4). There were 31 CalS orthologous gene pairs among $G$. arboretum and two allotetraploid cotton species, including 15 pairs between with At-subgenome of G. hirsutum and 16 pairs between with At-subgenome of G. barbadense. There were $17 \mathrm{CalS}$ orthologous gene pairs among G. raimondii and two allotetraploid cotton species, including 9 pairs between with Dt-subgenome of G. hirsutum and 8 pairs between with Dt-subgenome of $G$. barbadense. Meanwile, $\mathrm{Ka} / \mathrm{Ks}$ of CalS homologous pairs were calculated to further understand the adaptation of the CDS region of CalSs (Fig. 4, Table S5). Most of the homologous gene pairs $\mathrm{Ka} / \mathrm{Ks}<1$, and about $94.6 \%$ gene pairs had a $\mathrm{Ka} / \mathrm{Ks}$ ratio less than 0.5 , which meant that almost all gene pairs underwent purification selection. Only Ka/Ks $>1$ of GaCalS2 / GbCalS1 indicated that this was a positive selection for beneficial mutations.

\section{Analysis of Cis-acting elements in promoter.}

Transcription factors can be combined with cis-elements in the promoter region to regulate gene transcription. Investigation of upstream regulatory sequence can help us to well understand the regulation mechanism and also supportive to estimate the potential function of the gene (Fig. 5, Table S6). Given the effect of plant hormones in abiotic stress, we focused on plant hormone responsive elements in promoter regions. ABA- (ABRE), auxin- (AuxRR-core, TGA-element), Gibberellin- (GARE-motif, P-box, TATC-box), MeJA- (CGTCA-motif, TGACG-motif), SA(TCA-element) responsive element were found in the promoters of 18, 6, 14, 18, 12 GhCalSs. All GhCalSs contained hormone response elements except the GhCalSs in Group D and GhCalS5 in Group C. More than half of the GhCalSs contained ABA/GA/MeJA responsive element. Auxin responsive element only in GhCalS6/14/20/21/22/23. Meanwhile, we also paid attention to elements related to stress. Low-temperature- (LTR), wound- (WUN-motif), drought(MBS), stress- (TC-rich repeats), anaerobic induced response element (ARE), anoxic specific inducibility element (GC-motif) were found in the promoters of 11, 2, 15, 10, 23, 4 GhCalSs. Wound-responsive elements only in GhCalS5 and GhCalS18. Anoxic specific inducibility element only in GhCalS11/15/17/25. In addition, these results suggested that CalSs might regulated by hormone and abiotic stresses.

\section{Expression patterns of the GhCalSs under abiotic stresses.}

Previous studies have reported that the CalSs respond to abiotic stresses (Cui and Lee 2016; Jacobs AK; Fromm et al., 2013). To understand the response of GhCalSs, we used public RNAseq data of TM-1 treated with cold, hot, $\mathrm{NaCl}$, and PEG to observe the expression pattern of GhCalSs (Fig. 6). Interestingly, all expressed GhCalSs were induced by different abiotic stress, and the expression patterns were different. The expression of GhCalS3 was significantly upregulated under cold, hot, $\mathrm{NaCl}$, and PEG. The expression patterns of GhCalSs in the same group

PeerJ reviewing PDF | (2021:08:65037:2:0:NEW 27 Oct 2021) 
241 were slightly consistent, such as GhCalS3 and GhCalS6, GhCalS2 and GhCalS16. In order to

242 verify the results obtained by the above transcriptome, cotton seedlings were treated with PEG

243 and $\mathrm{NaCl}$, and then the GhCalS2/3/6/9/16 were selected for qRT-PCR (Fig. 7). The expression of

244 GhCalS3 and GhCalS6 in Group A were up-regulated within 24 hours under PEG treatment and

245 reached the peak at 24 hours. The expression of GhCalS2, GhCalS9, GhCalS16 were up-

246 regulated at first and then down-regulated and last up-regulated after PEG treatment. After $\mathrm{NaCl}$

247 treatment, there was no consistent trend of gene expression. GhCalS3 was significantly induced

248 by $\mathrm{NaCl}$ and significantly up-regulated at $3 \mathrm{~h}$. Both GhCalS2 and GhCalS16 of Group B were

249 down-regulated within 24 hours. The expression of GhCalS6 and GhCalS9 reached a peak at 12

250 h. These findings indicated that the expression patterns of several GhCalSs were changed after

251 treatment, which proved that GhCalSs increased adaptability to abiotic stress (Fig. 6).

252 Enrichment analysis of the GhCalSs.

253 In order to further understand the function of GhCalSs, we carried out functional enrichment annotation of gene ontology (GO) using pvalue of $\leq 0.05$ as the cutoff. The results improve our accurate understanding of gene function, including many significantly enriched terms (Fig8, S7 Table). The GO-BP enrichment results showed 34 terms such as (1->3)-beta-D-glucan biosynthetic process (GO:0006075), beta-glucan metabolic process (GO:0051273), cellular carbohydrate biosynthetic process (GO:0034637), cellular macromolecule biosynthetic process (GO:0034645). The GO-CC enrichment results discovered 16 terms such as 1,3-beta-D-glucan synthase complex (GO:0000148), plasma membrane protein complex (GO:0098797), transferase complex (GO:1990234), catalytic complex (GO:1902494). The CC terms enriched by GO were consistent with the subcellular localization of GhCalSs. GO-MF enrichment exposed 8 terms, including 1,3-beta-D-glucan synthase activity (GO:0003843), UDP-glucosyltransferase activity (GO:0035251), catalytic activity (GO:0003824), hexosyltransferase activity (GO:0016758). In short, the GO enrichment results confirmed the function of the GhCalSs in many biological processes, which were associated with 1,3- $\beta$-D-glucan synthetic activity, hydrolyzase activity, and membrane parts.

\section{GhCalSs expression patterns in various tissues and their role in fiber development.}

We used transcriptome data from different tissues GhCalSs to gain insight the tissue-specific expression patterns of cotton. For instance, GhCalSs were expressed in various tissues, and some of them were highly expressed. Some of GhCalSs were expressed in one or more tissues (GhCalS2, 3, 4, 6, 8, 9, 15, 16, 19, 20, 21). However, the expression of a few genes $(\mathrm{GhCalS1}, 5$, $7,10,11,12,13,14,17,18,22,23,24,25,26,27)$ did not show any expression in any tissues.

In order to determine the effect of GhCalSs in cotton fiber development, we focused on the expression of GhCalSs in different fiber development stages of two samples, MBI7747 and CCRI45, with different lengths and strengths (Lu et al., 2017) (Fig. 9). The expression of GhCalS4 was the highest in TM-1, MBI7747, CCRI45 fiber tissue, so it was speculated that GhCalS4 had an important function in cotton fiber development. In order to further determine its function in fiber development, qRT-PCR was used to analyze the GhCalS4 expression differences in two samples (Fig. 9). The results showed that the expression level of GhCalS4 in the two samples gradually increased from 5 DPA to 25 DPA, which was consistent with the 
282

283

284

285

286

287

288

289

290

291

292

293

294

295

296

297

298

299

300

301

302

303

304

305

306

307

308

309

310

311

312

313

314

315

316

317

318

319

320

321

transcriptome data of TM-1 used above. The expression of GhCalS4 in CCRI45 was higher than in MBI7747 at 5DPA, 10DPA, 15DPA but was significantly lower than that of MBI7747 at 25DPA (Lu et al., 2017). Thus, GhCalS4 may be involved in cotton fiber elongation.

\section{Discussion}

Callose plays a vital role in plant growth, development, and resistance to various adverse factors (Piršelová \& Matušíková 2012). The gene family of callose synthase has been identified in a variety of plants. In this study, we identified the CalSs in G. hirsutum, G. barbadense, $G$. raimondii, and $G$. arboreum, aiming to understand the role of CalS family in the cotton development.

A total of 86 CalSs were identified in four cotton species. They were divided into five groups based on evolutionary relationships. we divided CalSs (AtCalS9-12 homologous) into two groups due to the large difference of the CDS number, and the other groups were the same as those in Arabidopsis thaliana. The CalSs number was 2:2:1:1 in group B/C/D, which was consistent with the evolutionary relationship among cotton species (Table S3). Compared with Arabidopsis thaliana, different percentages existed between subgroups. The percentages of Group A and Group E were significantly different from those of the corresponding CalSs in Arabidopsis thaliana, suggesting that these genes in cotton may have functional differences with homologous genes in Arabidopsis thaliana to a certain extent. These results will help to validate the function of cotton CalS homologous gene with Arabidopsis thaliana.

Tetraploid cotton species are formed by natural crossbreeding between $G$. raimondii and $G$. arboretum (Wendel et al., 2009). Thus, the four cotton species are closely related in evolution. In Figure 4, the orthologous gene pairs of CalSs were all clustered in the same branch or group. Phylogenetic and orthologous genes of CalS further indicated that the results of this study were consistent with the evolutionary view.

A large number of hormone-responsive elements were identified on GhCalSs promoters which may be involved in the regulation of GhCalSs. Salicylic acid (SA) was an endogenous signal molecule in plants (Loake \& Grant, 2007). In Arabidopsis thaliana, the expressions of AtCalS1/5/9/10/12 were up-regulated by exogenous SA. Abscisic acid (ABA) played an important part in coping with a variety of adverse factors, closely related to callose synthesis (Liu et al., 2017). During the dormancy of Populus tomentosa buds, short-day induced ABA biosynthesis, promoted the expression of PtCalS1, callose deposited at the plasmodesmata to form blockage, which prevented the growth signal molecules from entering the cell and kept the dormancy state of buds (Tylewicz et al., 2018). Jasmonic acid (JA) was also involved in callose regulation, and Methyl Jasmonate (MeJA) application promoted callose deposition in grape leaves. Inhibition of the expression of Cationic peroxidase 3 (OCP3), a negative regulator of the JA pathway, increased callose deposition (Repka, Fischerová \& Šilhárová, 2004). In conclusion, ABA, JA, and SA were involved in the regulation of callose deposition. GhCalSs promoters with ABA, SA and JA response elements were highly likely to be regulated by them in cotton. However, how CalS gene is regulated by these hormones in the face of biotic-abiotic stress or growth and development is not known, which needs to be further studied. 
322

323

324

325

326

327

328

329

330

331

332

333

334

335

336

337

338

339

340

341

342

343

344

345

346

347

348

349

350

351

352

353

354

355

356

357

358

359

360

361

Callose deposition is one of a series of coping strategies in plants to abiotic stress. Low temperature stimulation of maize leaves increased callose content and reduced transport of photosynthate in phloem (Wu et al., 2018). In Arabidopsis thaliana, AtCalS7, AtCalS8 and AtCalS12 were associated with callose synthesis under the condition of wound (Jacobs et al., 2003; Cui and Lee 2016; Xie et al., 2011). In this study, public transcriptome data were used to analyze the responses of cotton leaves to cold, heat, salt and drought, and qRT-PCR was used to verify the results, which showed that CalSs were involved in abiotic stresses.

Callose deposits regulate material transport and control plant development. In this study, GhCalS4 was highly expressed in fibers and differentially expressed in MBI7747 and CCRI45 fibers at each fiber development stage (5/10/15/20/25 DPA). It has been reported that callose deposition may be involved in the closure of plasmodesmata, and the closure of plasmodesmata has an important function in the elongation of cotton fibers (Ruan et al., 2004). In Island cotton, plasmodesmata remain open longer than in Upland cotton, allowing sucrose to be fed into fibroblasts, which eventually increase osmotic potential by hydrolysis to fructose and glucose. The more soluble sugar, $\mathrm{K}^{+}$accumulated, the higher the cell leavening pressure, which promoted the elongation of cotton fiber (Hu et al., 2019). MBI7747 is a chromosome segment substitution line (CSSL) with different genetic background constructed by crosses between the upland cotton CCRI45 as the recurrent parent and the Sea Island cotton Hai 1 with outstanding fiber quality through the combination of high-generation backcrossing and molecular marker-assisted selection. The fiber length and strength of MBI7747 are better than CCRI45. In CCRI45, the expression level of this gene at 5DPA, 10DPA and 15DPA were all higher than those of MBI7747 during fiber elongation, and it was speculated that the degree of callose deposition in MBI7747 was lower than that of CCRI45, which made more sucrose input into fiber cells to increase osmotic potential and promote fiber elongation. Thus, GhCalS4 may be an introgression gene or there was difference in epigenetic regulation.

\section{Conclusions}

In this study, we identified 86 CalSs from G. hirsutum, G. barbadense, G. raimondii, and G. arboreum using conserved domains. Phylogeny, gene structure, motif, chromosome location and homologous genes were analyzed. It indicates that CalSs have been highly conserved during evolution by the analysis of CalSs structure, conversed motifs, and syntenic blocks.

WGD/Segmental replication was the main driving force for the amplification of CalS family in cotton, and purification selection played an important role in the evolution of CalSs. In addition, the cis-acting elements of GhCalSs related to hormone regulation and development and its expression pattern in stress and tissue were also analyzed. CalS gene can be induced by abiotic stress. Some genes in Group A may have the important function in the development of cotton tissues. Furthermore, the expression difference of GhCalS4 in fiber of different length and strength materials was analyzed. It was speculated that GhCalS4 played a major role in fiber elongation. These findings could lay a foundation for further study on the role of CalS gene in stress response and fiber development.

\section{References}


362

363

364

365

366

367

368

369

370

371

372

373

374

375

376

377

378

379

380

381

382

383

384

385

386

387

388

389

390

391

392

393

394

395

396

397

398

399

400

401

402

Ahmad S, Veyrat N, Gordon-Weeks R, Zhang Y, Martin J, Smart L, Glauser G, Erb M, Flors V, Frey M, Ton J. 2011. Benzoxazinoid metabolites regulate innate immunity against aphids and fungi in maize. Plant Physiology 157 (1):317-327. DOI 10.1104/pp.111.180224

Blümke A, Somerville SC, Voigt CA. 2013. Transient expression of the Arabidopsis thaliana callose synthase PMR4 increases penetration resistance to powdery mildew in barley. Advances in Bioscience and Biotechnology 04 (08):810-813. DOI 10.4236/abb.2013.48106

Chen C, Chen H, Zhang Y, Thomas HR, Frank MH, He Y, Xia R. 2020. TBtools: An Integrative Toolkit Developed for Interactive Analyses of Big Biological Data. Molecular Plant 13 (8): 1194-1202. DOI 10.1016/j.molp.2020.06.009

Chen XY, Kim JY. 2009. Callose synthesis in higher plants. Plant Signaling and Behavior. 4 (6):489-492. DOI 10.4161/psb.4.6.8359

Conant GC, Wolfe KH. 2008. Turning a hobby into a job: how duplicated genes find new functions. Nature Reviews Genetics 9 (12):938-950. DOI 10.1038/nrg2482

Cui W, Lee JY. 2016. Arabidopsis callose synthases CalS1/8 regulate plasmodesmal permeability during stress. Nature Plants 2 (5):16034. DOI 10.1038/nplants.2016.34

Cui X, Shin H, Song C, Laosinchai W, Amano Y, Brown RM, Jr. 2001. A putative plant homolog of the yeast beta-1,3-glucan synthase subunit FKS1 from cotton (Gossypium hirsutum L.) fibers. Planta 213 (2):223-230. DOI 10.1007/s004250000496

Dong X, Hong Z, Chatterjee J, Kim S, Verma DP. 2008. Expression of callose synthase genes and its connection with Npr1 signaling pathway during pathogen infection. Planta 229 (1):8798. DOI 10.1007/s00425-008-0812-3

Du X, Huang G, He S, Yang Z, Sun G, Ma X, Li N, Zhang X, Sun J, Liu M, Jia Y, Pan Z, Gong W, Liu Z, Zhu H, Ma L, Liu F, Yang D, Wang F, Fan W, Gong Q, Peng Z, Wang L, Wang X, Xu S, Shang H, Lu C, Zheng H, Huang S, Lin T, Zhu Y, Li F. 2018. Resequencing of 243 diploid cotton accessions based on an updated A genome identifies the genetic basis of key agronomic traits. Nature Genetics 50 (6):796-802. DOI 10.1038/s41588018-0116-X

Ellinger D, Glockner A, Koch J, Naumann M, Sturtz V, Schutt K, Manisseri C, Somerville SC, Voigt CA. 2014. Interaction of the Arabidopsis GTPase RabA4c with its effector PMR4 results in complete penetration resistance to powdery mildew. Plant Cell 26 (7):3185-3200. DOI 10.1105/tpc.114.127779

Ellinger D, Naumann M, Falter C, Zwikowics C, Jamrow T, Manisseri C, Somerville SC, Voigt CA. 2013. Elevated early callose deposition results in complete penetration resistance to powdery mildew in Arabidopsis. Plant Physiology 161 (3):1433-1444. DOI 10.1104/pp.112.211011

Enrique R, Siciliano F, Favaro MA, Gerhardt N, Roeschlin R, Rigano L, Sendin L, Castagnaro A, Vojnov A, Marano MR. 2011. Novel demonstration of RNAi in citrus reveals importance of citrus callose synthase in defence against Xanthomonas citri subsp. citri. Plant Biotechnology Journal 9 (3):394-407. DOI 10.1111/j.1467-7652.2010.00555.x

Fang L, Tian R, Li X, Chen J, Wang S, Wang P, Zhang T. 2014. Cotton fiber elongation network revealed by expression profiling of longer fiber lines introgressed with different

Peer) reviewing PDF | (2021:08:65037:2:0:NEW 27 Oct 2021) 
403

404

405

406

407

408

409

410

411

412

413

414

415

416

417

418

419

420

421

422

423

424

425

426

427

428

429

430

431

432

433

434

435

436

437

438

439

440

441

442

443

Gossypium barbadense chromosome segments. BMC Genomics 15:838. DOI https://doi.org/10.1186/1471-2164-15-838

Fromm J, Hajirezaei MR, Becker VK, Lautner S. 2013. Electrical signaling along the phloem and its physiological responses in the maize leaf. Frontiers in Plant Science 4:239. DOI 10.3389/fpls.2013.00239

Granato LM, Galdeano DM, D’Alessandre NDR, Breton MC, Machado MA. 2019. Callose synthase family genes plays an important role in the Citrus defense response to Candidatus Liberibacter asiaticus. European Journal of Plant Pathology 155 (1):25-38. DOI 10.1007/s10658-019-01747-6

Gu Z, Eils R, Schlesner M. 2016. Complex heatmaps reveal patterns and correlations in multidimensional genomic data. Bioinformatics 32 (18):2847-2849. DOI 10.1093/bioinformatics/btw313

He Z, Zhang H, Gao S, Lercher MJ, Chen WH, Hu S. 2016. Evolview v2: an online visualization and management tool for customized and annotated phylogenetic trees. Nucleic Acids Research 44 (W1):W236-241. DOI 10.1093/nar/gkw370

Hu Y, Chen J, Fang L, Zhang Z, Ma W, Niu Y, Ju L, Deng J, Zhao T, Lian J, Baruch K, Fang D, Liu X, Ruan YL, Rahman MU, Han J, Wang K, Wang Q, Wu H, Mei G, Zang Y, Han Z, Xu C, Shen W, Yang D, Si Z, Dai F, Zou L, Huang F, Bai Y, Zhang Y, Brodt A, Ben-Hamo H, Zhu X, Zhou B, Guan X, Zhu S, Chen X, Zhang T. 2019. Gossypium barbadense and Gossypium hirsutum genomes provide insights into the origin and evolution of allotetraploid cotton. Nature Genetics 51 (4):739-748. DOI 10.1038/s41588-019-0371-5

Huang L, Chen XY, Rim Y, Han X, Cho WK, Kim SW, Kim JY. 2009. Arabidopsis glucan synthase-like 10 functions in male gametogenesis. Journal of Plant Physiology 166 (4):344352. DOI 10.1016/j.jplph.2008.06.010

Jacobs AK, Lipka V, Burton RA, Panstruga R, Strizhov N, Schulze-Lefert P, Fincher GB. 2003. An Arabidopsis Callose Synthase, GSL5, Is Required for Wound and Papillary Callose Formation. Plant Cell 15 (11):2503-2513. DOI 10.1105/tpc.016097

Koh EJ, Zhou L, Williams DS, Park J, Ding N, Duan YP, Kang BH. 2012. Callose deposition in the phloem plasmodesmata and inhibition of phloem transport in citrus leaves infected with "Candidatus Liberibacter asiaticus". Protoplasma 249 (3):687-697. DOI 10.1007/s00709-011-0312-3

Kumar S, Stecher G, Tamura K. 2016. MEGA7: Molecular Evolutionary Genetics Analysis Version 7.0 for Bigger Datasets. Molecular Biology and Evolution 33 (7):1870-1874. DOI 10.1093/molbev/msw054

Lescot M, Déhais P, Thijs G, Marchal K, Moreau Y, Van de Peer Y, Rouzé P, Rombauts S. 2002. PlantCARE, a database of plant cis-acting regulatory elements and a portal to tools for in silico analysis of promoter sequences. Nucleic Acids Research 30(1):325-327. DOI 10.1093/nar/30.1.325

Liu F, Zou Z, Fernando WGD. 2018. Characterization of Callose Deposition and Analysis of the Callose Synthase Gene Family of Brassica napus in Response to Leptosphaeria maculans. International Journal of Molecular Sciences 19 (12). DOI 10.3390/ijms19123769 
444

445

446

447

448

449

450

451

452

453

454

455

456

457

458

459

460

461

462

463

464

465

466

467

468

469

470

471

472

473

474

475

476

477

478

479

480

481

482

483

Liu J, Du H, Ding X, Zhou Y, Xie P, Wu J. 2017. Mechanisms of callose deposition in rice regulated by exogenous abscisic acid and its involvement in rice resistance to Nilaparvata lugens Stal (Hemiptera: Delphacidae). Pest Management Science 73 (12):2559-2568. DOI $10.1002 / p s .4655$

Liu X, Zhao B, Zheng HJ, Hu Y, Lu G, Yang CQ, Chen JD, Chen JJ, Chen DY, Zhang L, Zhou Y, Wang LJ, Guo WZ, Bai YL, Ruan JX, Shangguan XX, Mao YB, Shan CM, Jiang JP, Zhu YQ, Jin L, Kang H, Chen ST, He XL, Wang R, Wang YZ, Chen J, Wang LJ, Yu ST, Wang BY, Wei J, Song SC, Lu XY, Gao ZC, Gu WY, Deng X, Ma D, Wang S, Liang WH, Fang L, Cai CP, Zhu XF, Zhou BL, Jeffrey Chen Z, Xu SH, Zhang YG, Wang SY, Zhang TZ, Zhao GP, Chen XY. 2015. Gossypium barbadense genome sequence provides insight into the evolution of extra-long staple fiber and specialized metabolites. Scientific Reports 5:14139. DOI 10.1038/srep14139

Livak KJ, Schmittgen TD. 2001. Analysis of relative gene expression data using real-time quantitative PCR and the 2(-Delta Delta C(T)) Method. Methods 25 (4):402-408. DOI 10.1006/meth.2001.1262

Loake G, Grant M. 2007. Salicylic acid in plant defence--the players and protagonists. Current Opinion in Plant Biology 10 (5):466-472. DOI 10.1016/j.pbi.2007.08.008

Lu Q, Shi Y, Xiao X, Li P, Gong J, Gong W, Liu A, Shang H, Li J, Ge Q, Song W, Li S, Zhang Z, Rashid MHO, Peng R, Yuan Y, Huang J. 2017. Transcriptome Analysis Suggests That Chromosome Introgression Fragments from Sea Island Cotton (Gossypium barbadense) Increase Fiber Strength in Upland Cotton (Gossypium hirsutum). G3-Genes Genomes Genetics 7 (10):3469-3479. DOI 10.1534/g3.117.300108

Magwanga RO, Lu P, Kirungu JN, Cai X, Zhou Z, Wang X, Diouf L, Xu Y, Hou Y, Hu Y, Dong Q, Wang K, Liu F. 2018. Whole Genome Analysis of Cyclin Dependent Kinase (CDK) Gene Family in Cotton and Functional Evaluation of the Role of CDKF4 Gene in Drought and Salt Stress Tolerance in Plants. International Journal of Molecular Sciences 19 (9):2625. DOI 10.3390/ijms19092625

Oide S, Bejai S, Staal J, Guan N, Kaliff M, Dixelius C. 2013. A novel role of PR2 in abscisic acid (ABA) mediated, pathogen-induced callose deposition in Arabidopsis thaliana. New Phytologist 200 (4):1187-1199. DOI 10.1111/nph.12436

Paterson AH, Wendel JF, Gundlach H, Guo H, Jenkins J, Jin D, Llewellyn D, Showmaker KC, Shu S, Udall J, Yoo MJ, Byers R, Chen W, Doron-Faigenboim A, Duke MV, Gong L, Grimwood J, Grover C, Grupp K, Hu G, Lee TH, Li J, Lin L, Liu T, Marler BS, Page JT, Roberts AW, Romanel E, Sanders WS, Szadkowski E, Tan X, Tang H, Xu C, Wang J, Wang Z, Zhang D, Zhang L, Ashrafi H, Bedon F, Bowers JE, Brubaker CL, Chee PW, Das S, Gingle AR, Haigler CH, Harker D, Hoffmann LV, Hovav R, Jones DC, Lemke C, Mansoor S, ur Rahman M, Rainville LN, Rambani A, Reddy UK, Rong JK, Saranga Y, Scheffler BE, Scheffler JA, Stelly DM, Triplett BA, Van Deynze A, Vaslin MF, Waghmare VN, Walford SA, Wright RJ, Zaki EA, Zhang T, Dennis ES, Mayer KF, Peterson DG, Rokhsar DS, Wang X, Schmutz J. 2012. Repeated polyploidization of 
Gossypium genomes and the evolution of spinnable cotton fibres. Nature 492 (7429):423-427. DOI 10.1038/nature11798

Piršelová B, Matušíková I. 2012. Callose: the plant cell wall polysaccharide with multiple biological functions. Acta Physiologiae Plantarum 35 (3):635-644. DOI 10.1007/s11738-0121103-y

Pu Y, Hou L, Guo Y, Ullah I, Yang Y, Yue Y. 2019. Genome-wide analysis of the callose enzyme families of fertile and sterile flower buds of the Chinese cabbage (Brassica rapa L. ssp. pekinensis). FEBS Open Bio 9 (8): 1432-1449. DOI 10.1002/2211-5463.12685

Repka V, Fischerová, I. \& Šilhárová, K. 2004. Methyl jasmonate is a potent elicitor of multiple defense responses in grapevine leaves and cell-suspension cultures. Biologia Plantarum 48:273-283 DOI https://doi.org/10.1023/B:BIOP.0000033456.27521.e5

Ruan YL, Xu SM, White R, Furbank RT. 2004. Genotypic and developmental evidence for the role of plasmodesmatal regulation in cotton fiber elongation mediated by callose turnover. Plant Physiology 136 (4):4104-4113. DOI 10.1104/pp.104.051540

Schober MS, Burton RA, Shirley NJ, Jacobs AK, Fincher GB. 2009. Analysis of the (1,3)beta-D-glucan synthase gene family of barley. Phytochemistry 70 (6):713-720. DOI 10.1016/j.phytochem.2009.04.002

Shi X, Sun X, Zhang Z, Feng D, Zhang Q, Han L, Wu J, Lu T. 2015. GLUCAN SYNTHASE-LIKE 5 (GSL5) plays an essential role in male fertility by regulating callose metabolism during microsporogenesis in rice. Plant Cell Physiology 56(3):497-509. DOI $10.1093 / \mathrm{pcp} / \mathrm{pcu} 193$

Shi ZH, Zhang C, Xu XF, Zhu J, Zhou Q, Ma LJ, Niu J, Yang ZN. 2015. Overexpression of AtTTP affects ARF17 expression and leads to male sterility in Arabidopsis. PLoS One 10 (3):e0117317. DOI 10.1371/journal.pone.0117317

Song L, Wang R, Zhang L, Wang Y, Yao S. 2016. CRR1 encoding callose synthase functions in ovary expansion by affecting vascular cell patterning in rice. Plant Journal 88 (4):620-632. DOI 10.1111/tpj.13287

Sun M, Voorrips RE, Steenhuis-Broers G, Van't Westende W, Vosman B. 2018. Reduced phloem uptake of Myzus persicae on an aphid resistant pepper accession. BMC Plant Biology 18 (1):138. DOI 10.1186/s12870-018-1340-3

Toller A, Brownfield L, Neu C, Twell D, Schulze-Lefert P. 2008. Dual function of Arabidopsis glucan synthase-like genes GSL8 and GSL10 in male gametophyte development and plant growth. Plant Journal 54 (5):911-923. DOI 10.1111/j.1365-313X.2008.03462.x

Tylewicz S, Petterle A, Marttila S, Miskolczi P, Azeez A, Singh RK, Immanen J, Mähler N, Hvidsten TR, Eklund DM, Bowman JL, Helariutta Y, Bhalerao RP. 2018. Photoperiodic control of seasonal growth is mediated by ABA acting on cell-cell communication. Science 360(6385):212-215. DOI 10.1126/science.aan8576

Wang X, Lu X, Malik WA, Chen X, Wang J, Wang D, Wang S, Chen C, Guo L, Ye W. 2020. Differentially expressed bZIP transcription factors confer multi-tolerances in Gossypium hirsutum L. International Journal of Biological Macromolecules. 146:569-578. DOI 10.1016/j.ijbiomac.2020.01.013 
525

526

527

528

529

530

531

532

533

534

535

536

537

538

539

540

541

542

Wang D, Zhang Y, Zhang Z, Zhu J, Yu J. 2010. KaKs_Calculator 2.0: A Toolkit

Incorporating Gamma-Series Methods and Sliding Window Strategies. Genomics, Proteomics \& Bioinformatics 8 (1):77-80. DOI 10.1016/s1672-0229(10)60008-3

Wendel JF, Brubaker C, Alvarez I, Cronn R, Stewart JM. 2009. Evolution and Natural History of the Cotton Genus. Genetics and Genomics of Cotton, 3-22. DOI 10.1007/978-0387-70810-2_1

Wu SW, Kumar R, Iswanto ABB, Kim JY. 2018. Callose balancing at plasmodesmata. Journal of Experimental Botany 69 (22):5325-5339. DOI 10.1093/jxb/ery317

Xie B, Wang X, Hong Z. 2010. Precocious pollen germination in Arabidopsis plants with altered callose deposition during microsporogenesis. Planta 231 (4):809-823. DOI 10.1007/s00425-009-1091-3

Xie B, Wang X, Zhu M, Zhang Z, Hong Z. 2011. CalS7 encodes a callose synthase responsible for callose deposition in the phloem. Plant Journal 65 (1):1-14. DOI 10.1111/j.1365313X.2010.04399.x

Yao L, Zhong Y, Wang B, Yan J, Wu T. 2020. BABA application improves soybean resistance to aphid through activation of phenylpropanoid metabolism and callose deposition. Pest Management Science 76 (1):384-394. DOI 10.1002/ps.5526

Yu CS, Chen YC, Lu CH, Hwang JK. 2006. Prediction of protein subcellular localization. Proteins 64 (3):643-651. DOI 10.1002/prot.21018

Yu J, Jung S, Cheng CH, Ficklin SP, Lee T, Zheng P, Jones D, Percy RG, Main D. 2014. CottonGen: a genomics, genetics and breeding database for cotton research. Nucleic Acids Research 42 (Database issue):D1229-1236. DOI 10.1093/nar/gkt1064

Yu Y, Jiao L, Fu S, Yin L, Zhang Y, Lu J. 2016. Callose Synthase Family Genes Involved in the Grapevine Defense Response to Downy Mildew Disease. Phytopathology 106 (1):56-64. DOI 10.1094/PHYTO-07-15-0166-R

Zavaliev R, Ueki S, Epel BL, Citovsky V. 2011. Biology of callose (beta-1,3-glucan) turnover at plasmodesmata. Protoplasma 248 (1):117-130. DOI 10.1007/s00709-010-0247-0

Hong Z, Delauney AJ, Verma DP. 2001. A cell plate-specific callose synthase and its interaction with phragmoplastin. Plant Cell. 13(4):755-68. DOI 10.1105/tpc.13.4.755. 
Figure 1

Figure 1. Phylogenetic analysis of CalS protein from G. hirsutum, G. barbadense, $G$. raimondii, $G$. arboretum and Arabidopsis.

The bootstrap values are shown at the nodes. The CalSs from G. hirsutum, G. barbadense, G. raimondii, G. arboretum, and Arabidopsis are marked with red check, orange rect, purple star, green triangle, grey circle, respectively. 


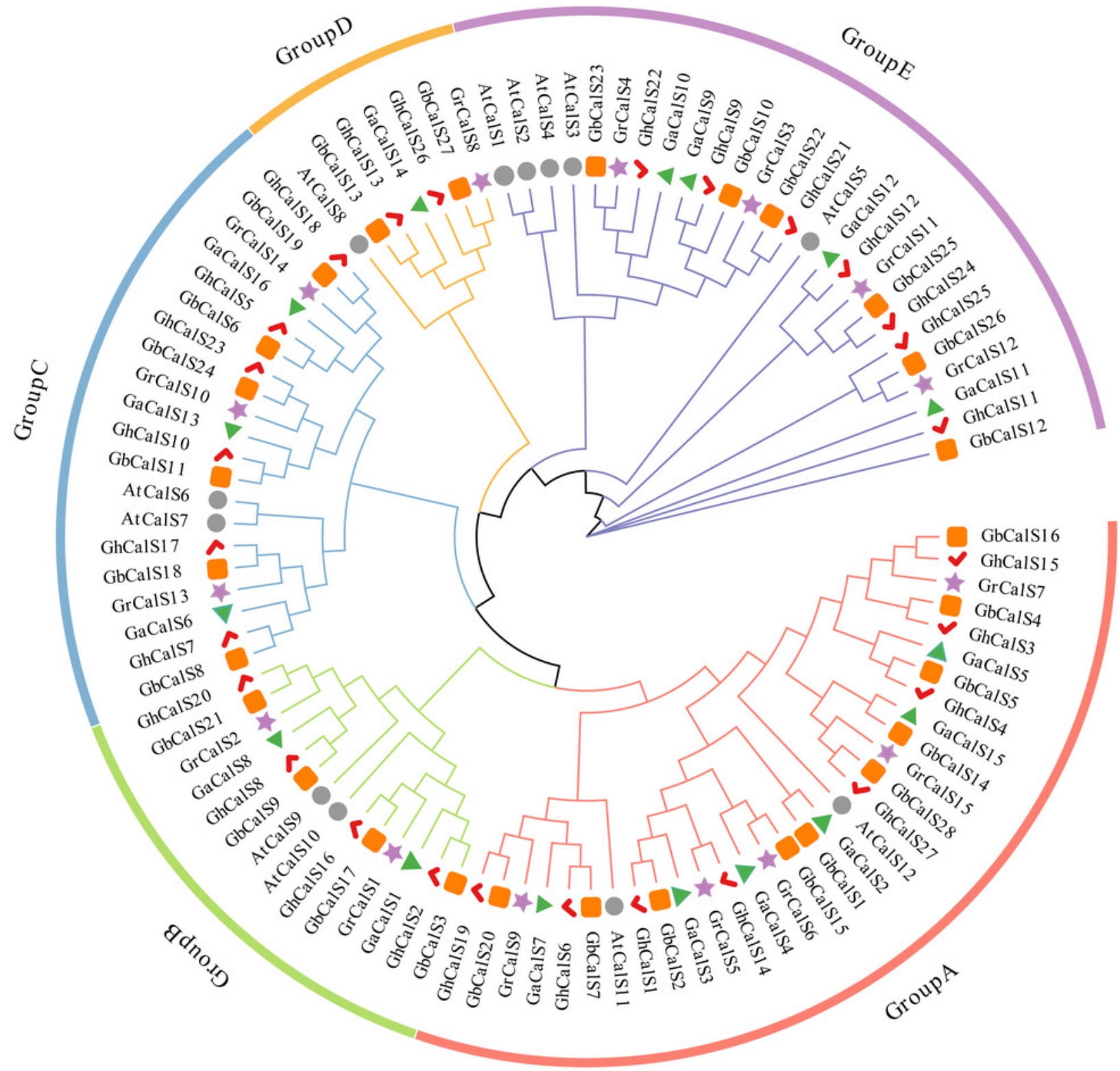


Figure 2

Conservative motif and exon-intron structure of CalS genes in cotton.

(A) The evolutionary tree of CalSs was constructed using MEGA7. (B) Conservative motif of CalSs. The 20 motifs are displayed in different colored boxes. (C) Exon-intron structure of CalSs. Introns are presented by grey lines, exons by green boxes, and UTR for yellow boxes.

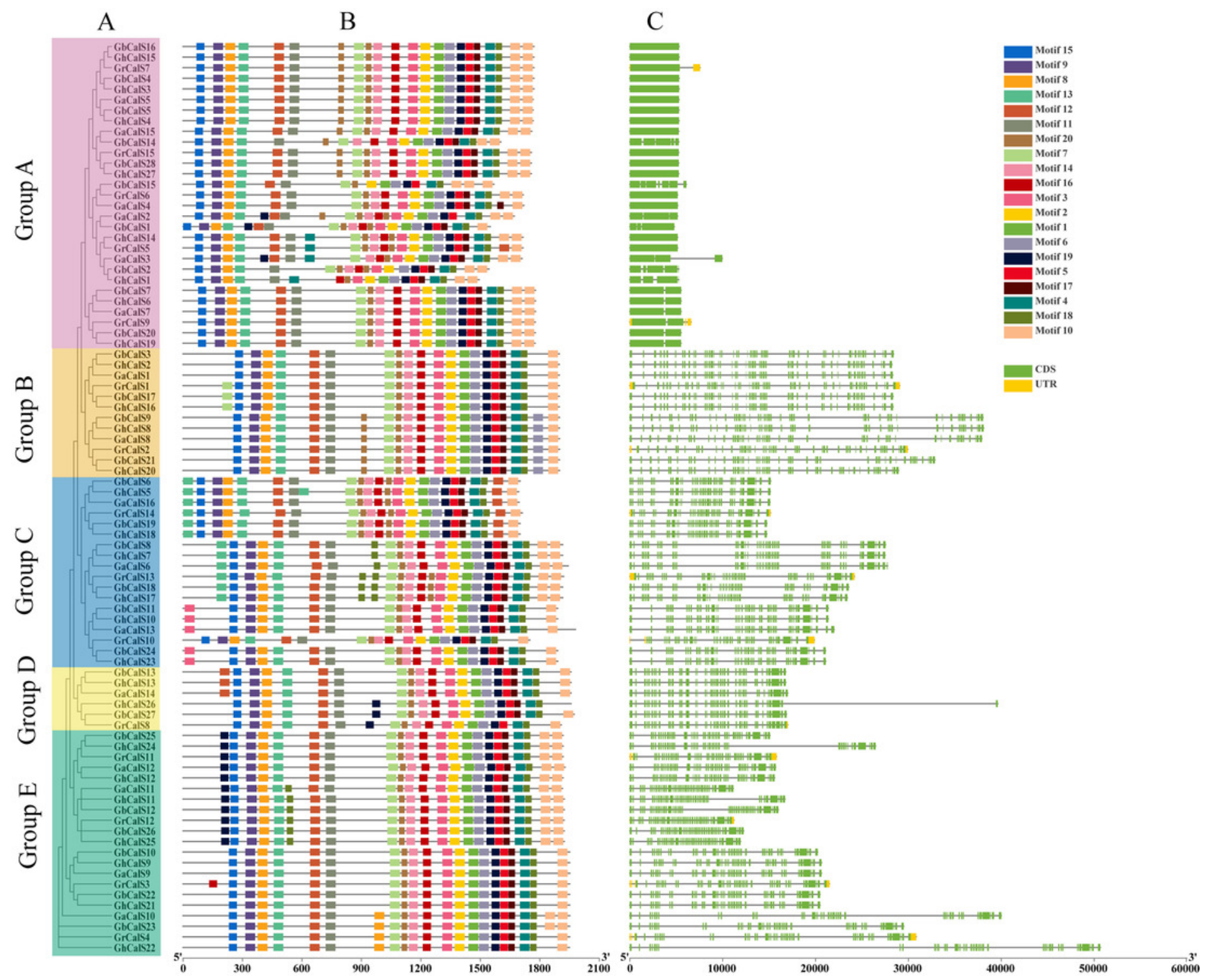


Figure 3

Figure 3. Distribution of 86 CalSs on Cotton Chromosomes.

The chromosome name is on the left of each chromosome, and the gene ID is on the right.

(A) G. hirsutum; (B) G. barbadense; (C) G. arboretum; (D) G. raimondii; 
A

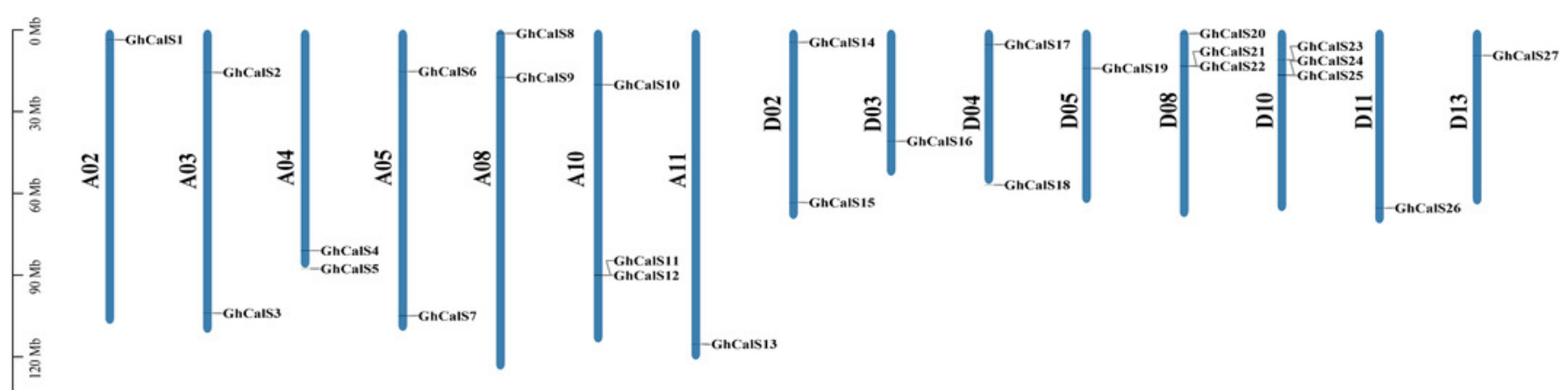

B

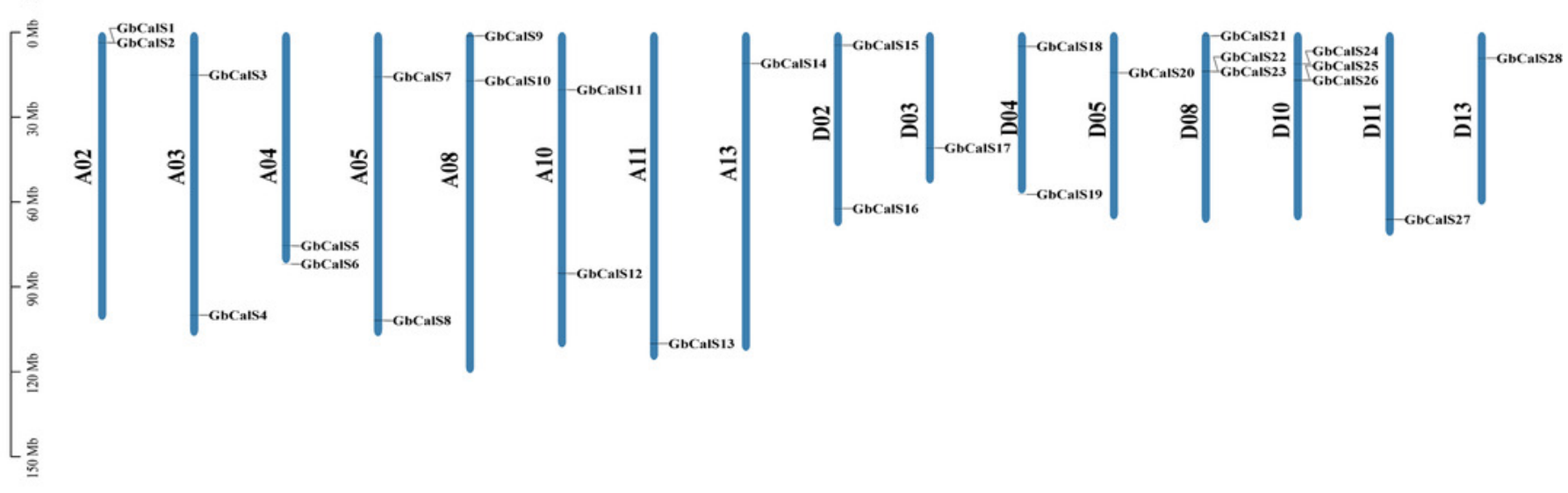

C

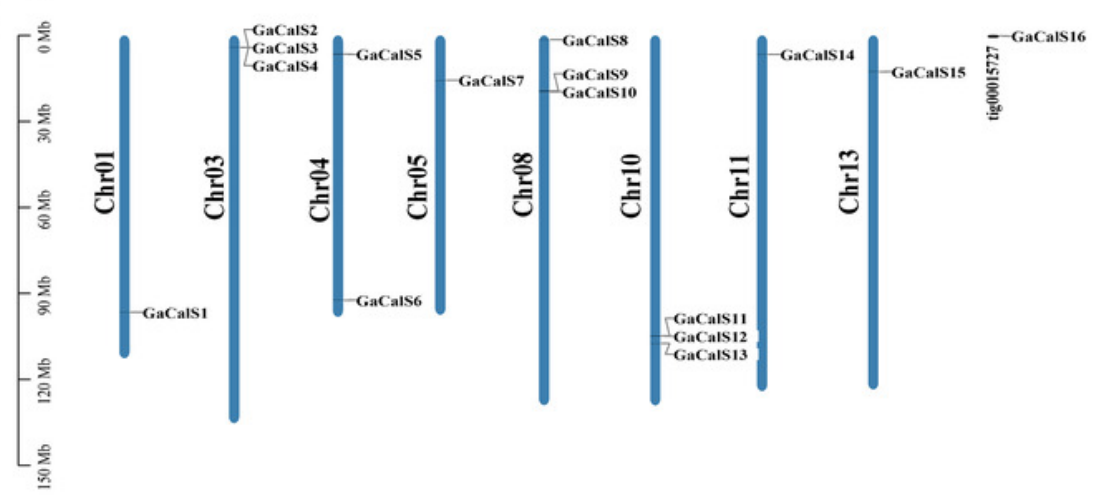

D

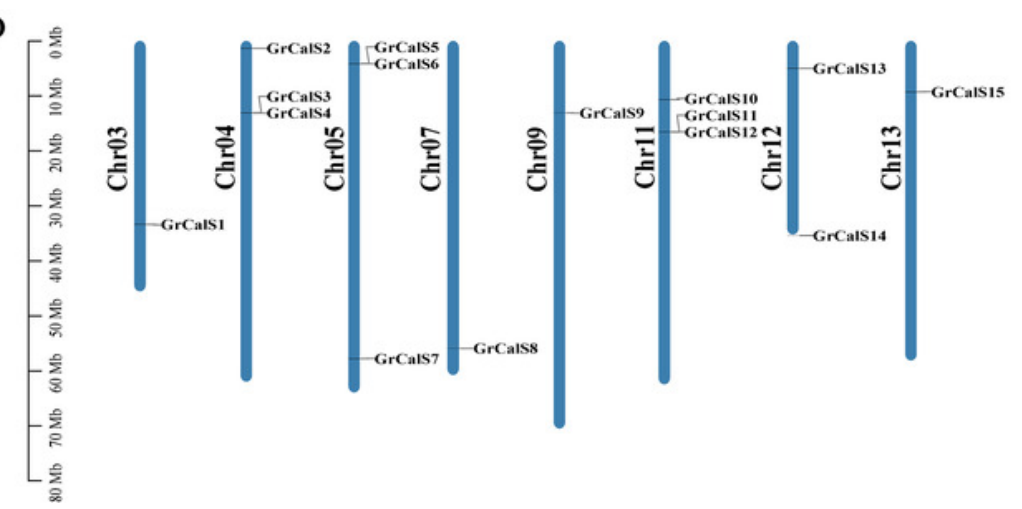


Figure 4

Collinearity analysis of CalSs in tetraploid and diploid cotton.

(A) Orthologous and paralogous gene pairs among tetraploid and diploid cotton species. The lines represented by various colors indicates the syntenic regions around CalSs, and the color between the same species is the same (B) Ka, Ks, Ka/Ks distribution of CalS gene pairs. Ka, Ks, Ka/Ks analysis of GbCalS-GaCalS, GbCalS-GbCalS, GbCalS-GhCalS, GbCalS-GrCalS, GhCalS-GaCalS, GhCalS-GhCalS, GhCalS-GrCalS.

A

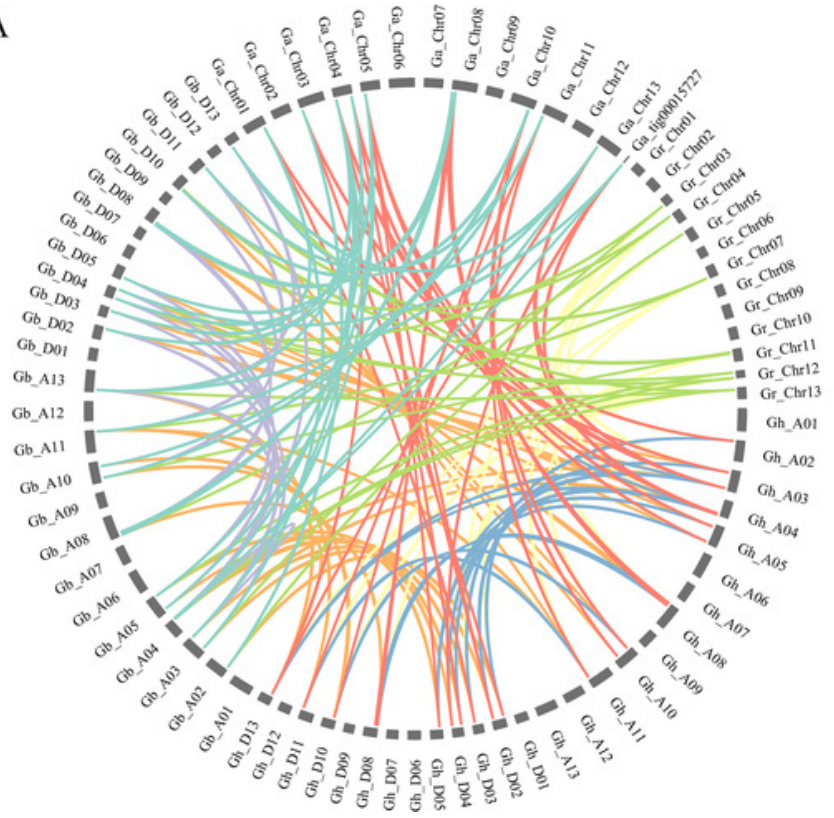

B
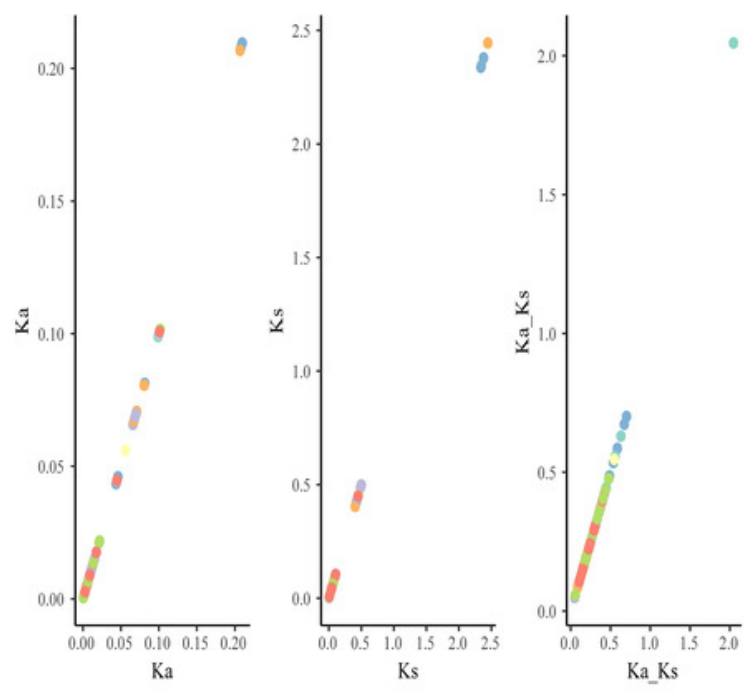

type

- GbCals Gacals

GbCal__bbcals

- GbCal_GhCals

- GbCals GrCals

- GhCals_Gacals

- GhCals_GhCals

- GhCals Gircals 
Figure 5

Cis-acting elements on promoters of the GhCalSs.

(A)The evolutionary tree of GhCalSs was constructed wsing MEGA7. (B) The cis-acting element on the promoter of GhCalSs. Number of each cis-acting element in the promoter region. 
A B

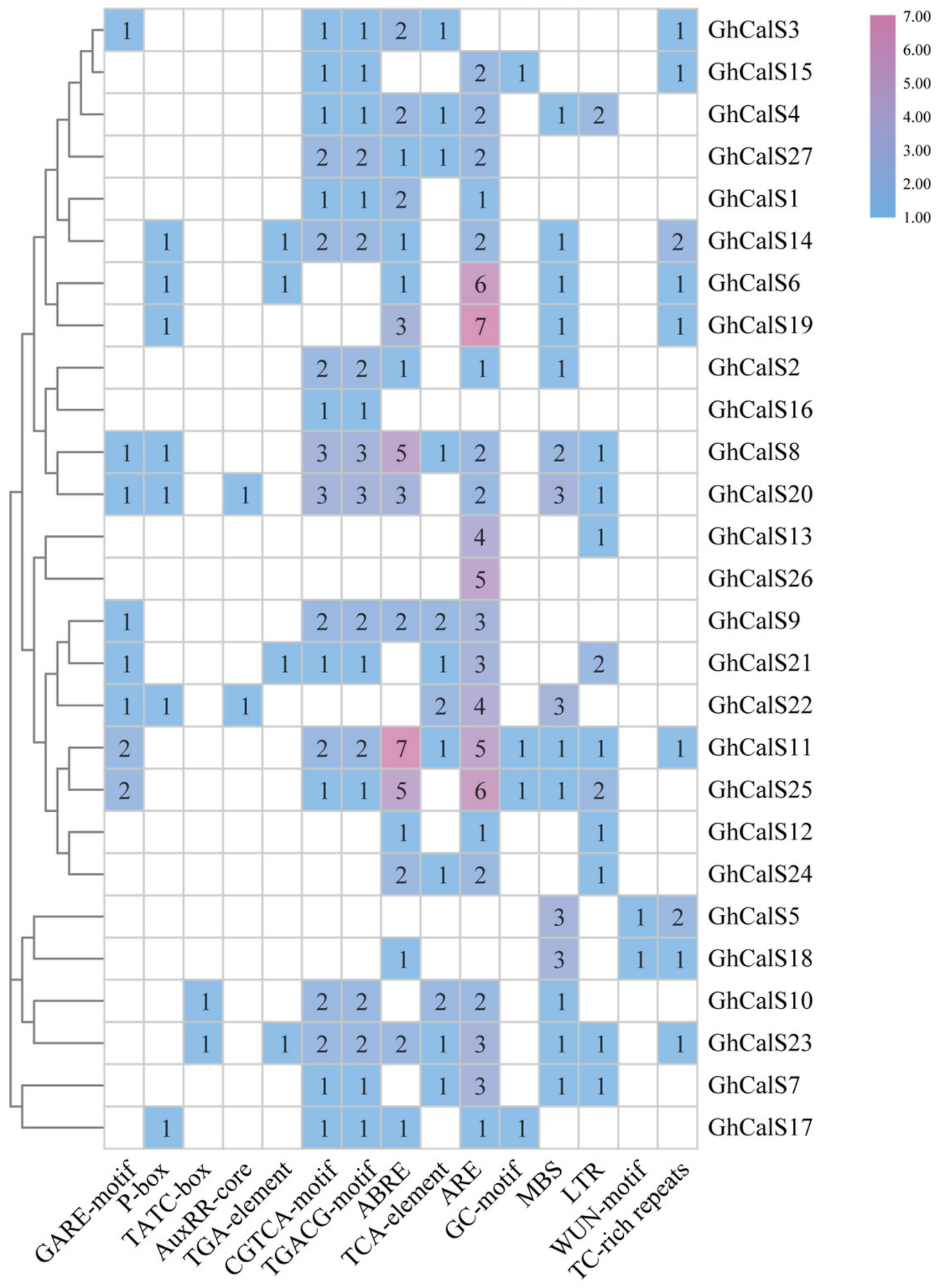


Figure 6

RNA sequence profiling of the CalS gene family.

(A) Heatmap displaying expression of expressed GhCalSs under hot, cold, $\mathrm{NaCl}$, and PEG treatment (B) Heatmap displaying expression of expressed GhCalSs in each tissue.

A

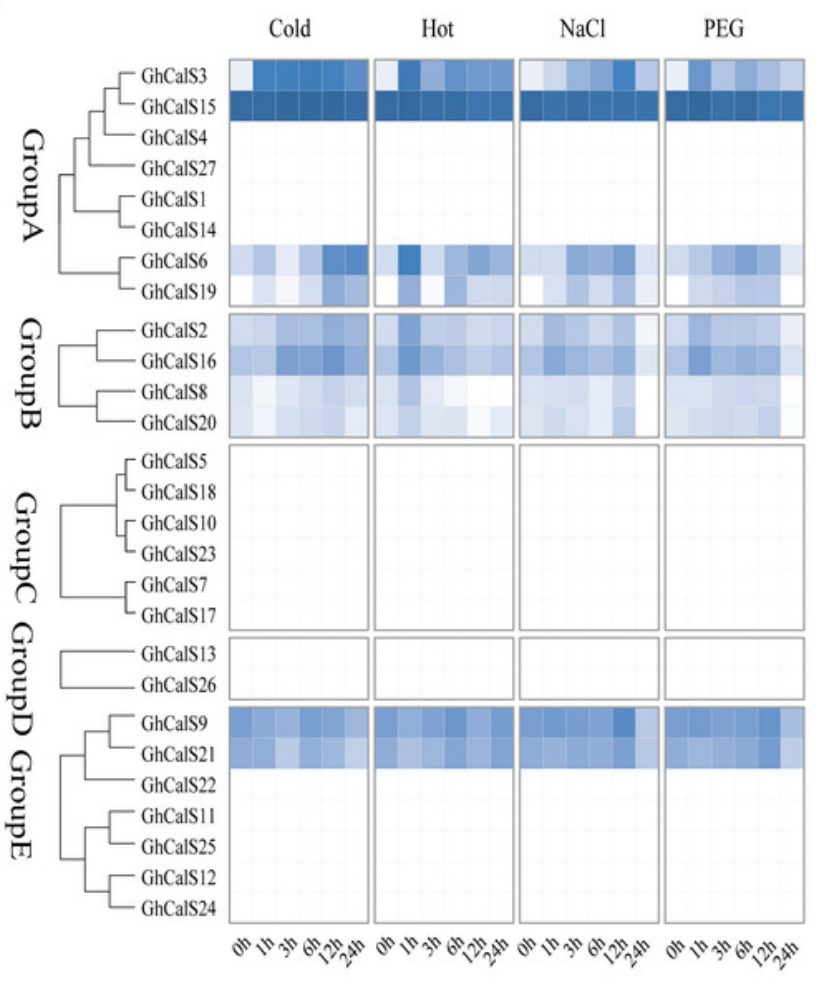

B

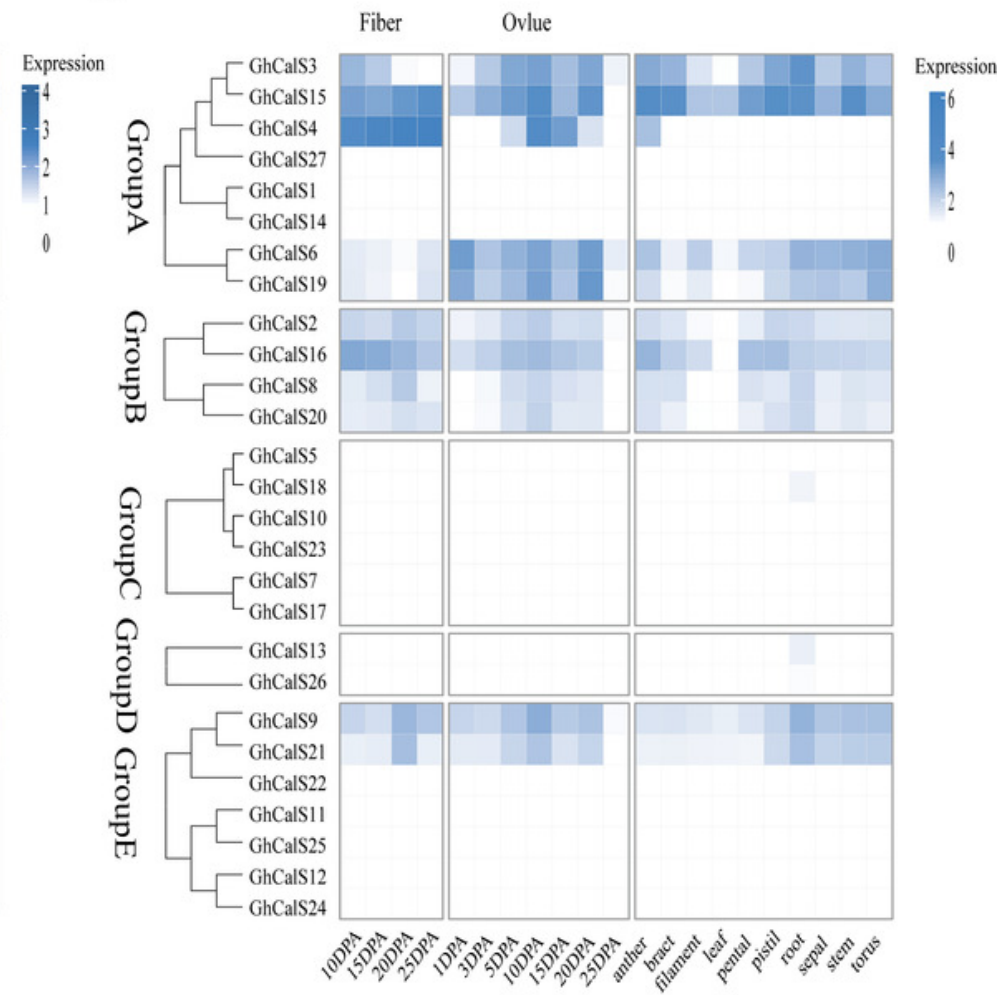


Figure 7

qRT-PCR results of GhCalSs under PEG and $\mathrm{NaCl}$.
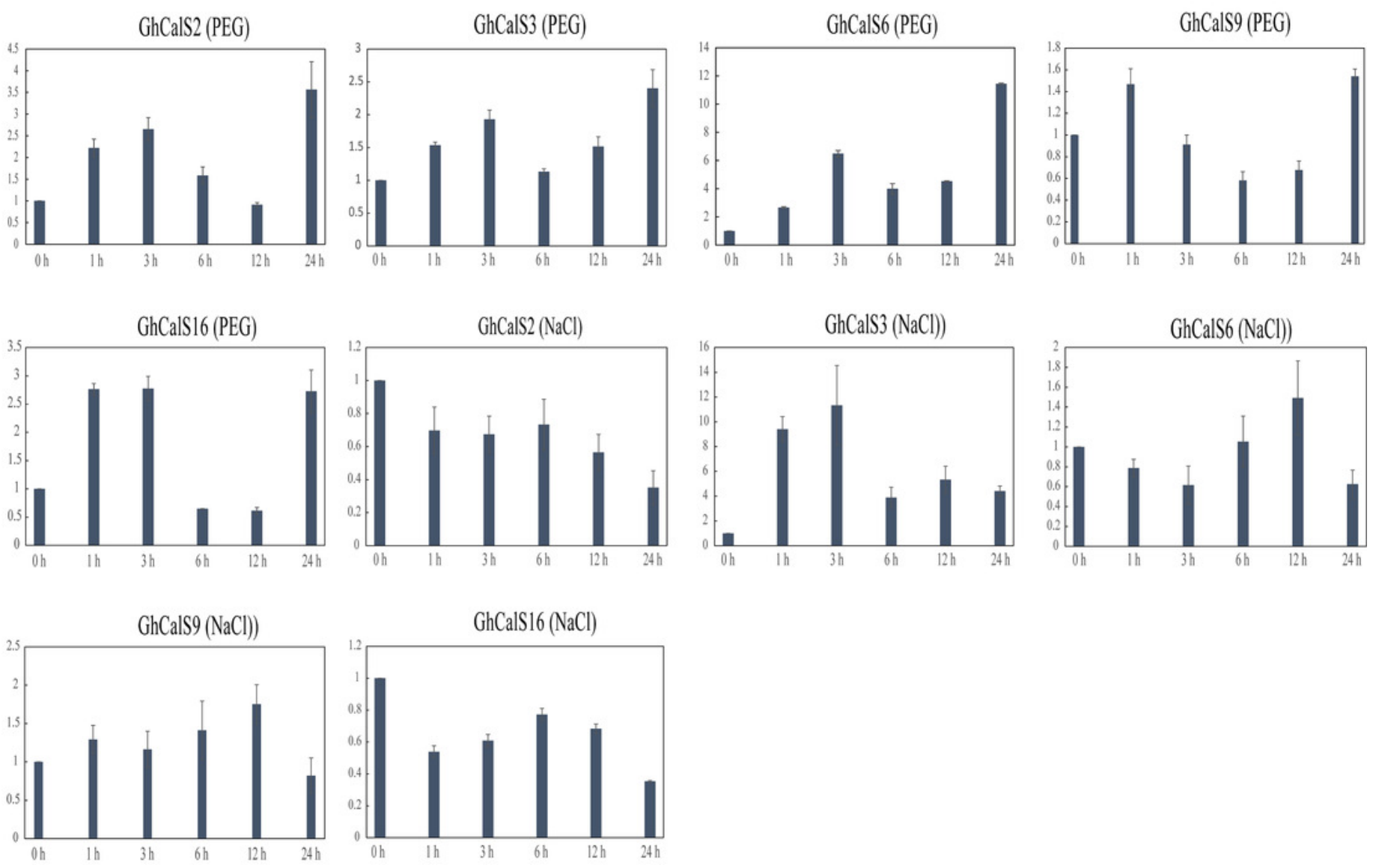


\section{Figure 8}

\section{Bubble plot showing GO enrichment analysis of GhCalSs.}

\section{The top 20 GO terms significantly enriched by GhCalSs.}

$$
\begin{array}{r}
\text { 1,3-beta-D-glucan synthase activity } \\
(1->3) \text { - beta-D-glucan biosynthetic process } \\
(1->3) \text {-beta-D-glucan metabolic process } \\
1,3 \text { - beta-D-glucan synthase complex } \\
\text { plasma membrane protein complex } \\
\text { beta-glucan biosynthetic process } \\
\text { beta-glucan metabolic process } \\
\text { glucan biosynthetic process } \\
\text { UDP-glucosyltransferase activity } \\
\text { glucosyltransferase activity } \\
\text { plasma membrane part }
\end{array}
$$
cellular polysaccharide biosynthetic process polysaccharide biosynthetic process cellular carbohydrate biosynthetic process cellular glucan metabolic process glucan metabolic process UDP-glycosyltransferase activity carbohydrate biosynthetic process cellular polysaccharide metabolic process transferase complex

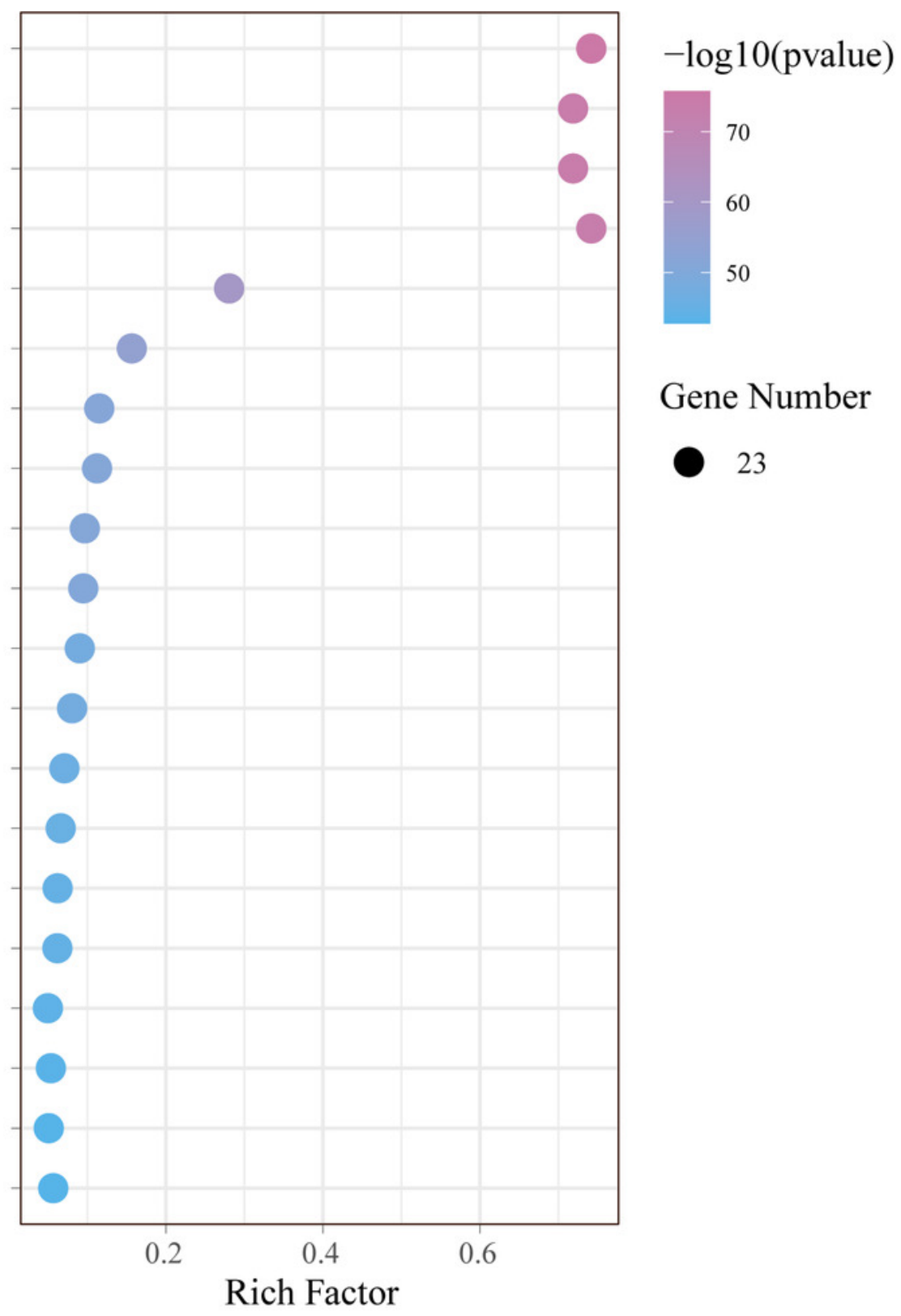


Figure 9

Expression patterns of GhCalSs in cotton fiber.

(A)The expression of GhCalSs of MBI7747 and CCRI45 at different fiber development stages.

(B) qRT-PCR results of GhCalS4 at different fiber development stages.

A

B

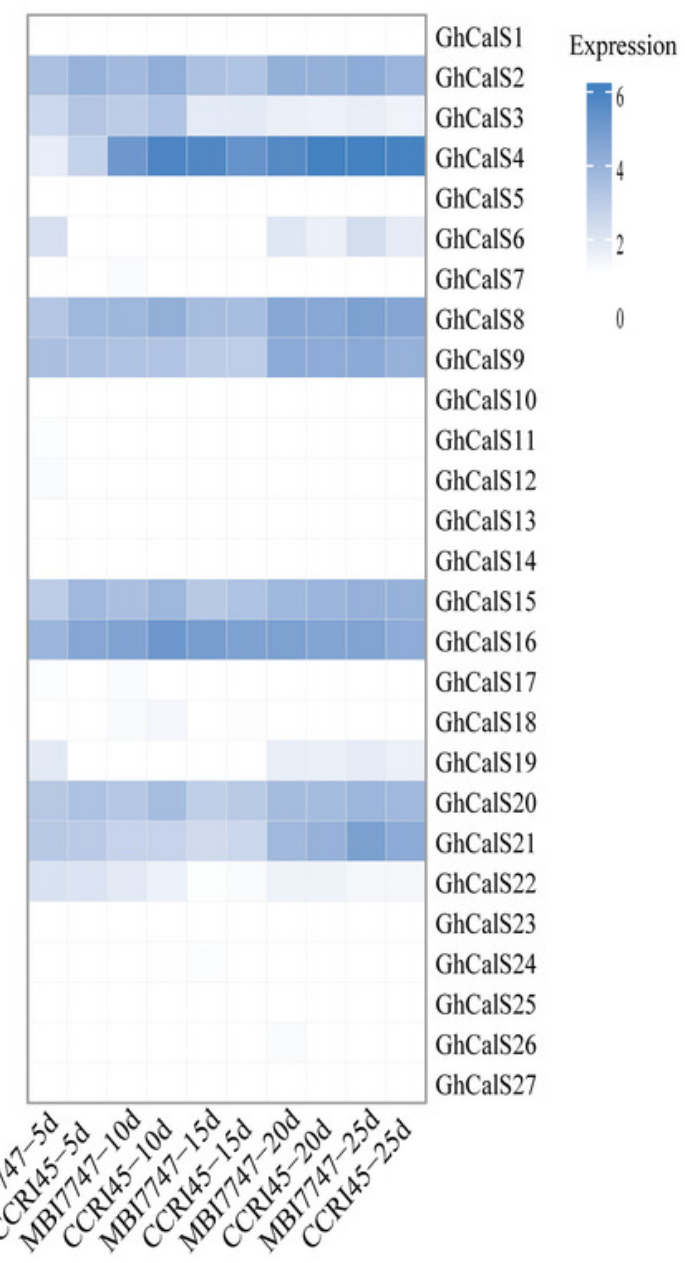

GhCalS4(fiber)

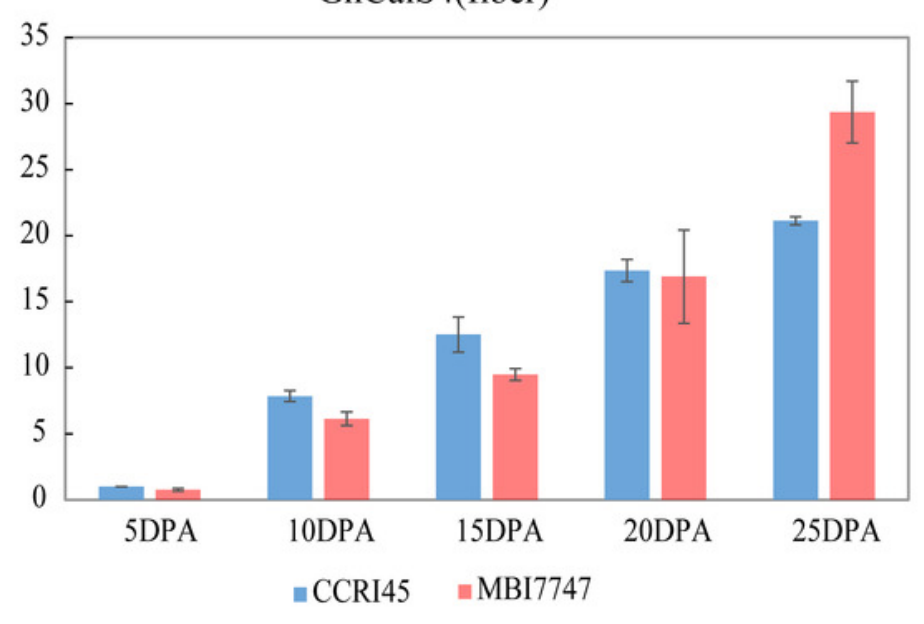

